\title{
Tapering and discontinuation of biologics in patients with psoriatic arthritis with low disease activity
}

Weiyu Ye ${ }^{1}$, Laura J. Tucker², Laura C. Coates ${ }^{2}$

1. Oxford University Clinical Academic Graduate School, University of Oxford, UK

2. Nuffield Department of Orthopaedics, Rheumatology and Musculoskeletal Sciences, University of Oxford, UK

Corresponding author:

Dr Weiyu Ye (ORCID: 0000-0002-8983-0355)

OUCAGS, Room 3A31, The Cairns Library IT Corridor, Level 3, John Radcliffe Hospital (Main Hospital)

Oxford, OX3 9DU

Email: christina.ye@medsci.ox.ac.uk

Tel: 01865289467

Word count: 3752

Running head: biologic tapering and discontinuation in psoriatic arthritis

\section{Compliance with ethical standards}

Funding: Weiyu Ye is an academic foundation trainee. Laura J Tucker is funded by the Norman Collisson Foundation as a Clinical Research Fellow. Laura C Coates is funded by a National Institute for Health Research Clinician Scientist award. The research was supported by the National Institute for Health Research (NIHR) Oxford Biomedical Research Centre (BRC). The views expressed are those of the author(s) and not necessarily those of the NHS, the NIHR or the Department of Health.

Conflict of interest: Laura C Coates has received research funding and/or honoraria from Abbvie, Amgen, BMS, Celgene, Galapagos, Janssen, Lilly, MSD, Novartis, Pfizer, Prothena and UCB. The other authors have no conflicts of interest to disclose. 


\section{Abstract}

The introduction of biologic disease modifying anti-rheumatic drugs (bMDARDs) have revolutionised the treatment of psoriatic arthritis (PsA). This, combined with a 'treat-to-target' approach, means that achieving remission is increasingly possible. In patients with well-controlled PsA, there is little consensus on whether bDMARDs should be continued, tapered or discontinued altogether. Tapering or discontinuation of bDMARDs could offer significant financial savings and minimise patient burden and unwanted drug-related side effects. However, there is a risk of loss of remission. This primary focus of this paper is to review the current evidence on bDMARD tapering and discontinuation in PSA. We explore the criteria employed by studies to define patients eligible for bDMARD tapering or discontinuation and the process by which this occurs. We also review the outcomes of bDMARD tapering and discontinuation, the predictors, and the likelihood of restoring remission following relapse. To date, bDMARD tapering seems to be feasible and safe in patients with PsA who are in remission or with low disease activity. Lower disease activity and increased duration on tumour necrosis factor inhibitors (TNFi) increases the likelihood of successful bDMARD tapering. In contrast, discontinuing bDMARDs appears to carry a substantial risk of loss of remission. Those with higher disease activity at time of TNFi discontinuation, current smokers, male sex, increased skin involvement, and synovial hypertrophy seen on ultrasound prior to discontinuation are at greater risk of losing remission post bDMARD discontinuation. In those who lose remission, reinstating the standard dose of bDMARD appears to be effective in restoring remission.

\section{Key points}

- In patients with well-controlled psoriatic arthritis on biologics, there is little consensus on whether they should continue on the same dose, taper the dose or discontinue the biologic altogether.

- Current evidence is based on small retrospective observational studies, and suggests that biologic tapering is preferable to discontinuation, which carries a substantial risk of loss of remission.

- There is a need for high-quality randomised controlled trials to substantiate current evidence in order to guide clinical decision making. If safe and feasible, tapering or discontinuation can result in substantial financial savings and also minimise treatment burden and drug-related side effects for patients. 


\section{Introduction}

Psoriatic arthritis (PSA) is an inflammatory spondyloarthritis characterised by arthritis, enthesitis, dactylitis and axial disease, in addition to skin and nail disease [1]. It can lead to progressive joint damage and loss of function, with a significant impact on patients' quality of life [2].

Current management of PsA aims to achieve disease control by preventing flares and inducing remission. Traditionally, a stepup approach is used starting with conventional synthetic disease-modifying anti-rheumatic drugs (csDMARDs) such as methotrexate, and escalating to a biologic disease-modifying anti-rheumatic drug (bDMARD) if there is an inadequate response $[3,4]$. Tumour necrosis factor inhibitors (TNFi) are the most commonly used bDMARDs in PSA, and have shown consistent efficacy in treating active disease and potentially halting peripheral joint structural damage [5]. A seminal study of 206 patients with early PSA randomised to either 'treat-to-target' or standard care found that tight control of disease activity significantly improved joint outcomes [6]. As a result of this, recent recommendations strongly advocate a 'treat-to-target' approach, whereby patients are regularly reviewed and treatment escalated if remission or minimal disease activity (MDA) criteria are not met. This, combined with the continually expanding repertoire of pharmacological therapies, means that remission or MDA states are increasingly achievable in PsA.

In patients with well-controlled disease, there is little consensus on whether they should be maintained on the same dose of bDMARDs, whether the dose should be tapered, or discontinued altogether. In resource-limited healthcare systems, tapering or discontinuation of bDMARDs could offer significant financial savings. For patients, it could minimise drug side effects including the risk of long-term immunosuppression. Reducing the frequency of drug administration could also minimise clinic and hospital visits, thereby reducing the treatment burden experienced by patients. However, dose-tapering does carry the risk of loss of disease control and disease relapse.

At present, most of the evidence on tapering or discontinuation of bDMARDs are derived from studies in rheumatoid arthritis (RA). Two recent reviews concluded that tapering bDMARDs is preferable to discontinuation in RA, as discontinuation carries a significant risk of loss of remission [7,8]. One meta-analysis found that tapering bDMARDs resulted in an increased risk of losing remission. However, there was no increased risk of radiographic progression or loss of low disease activity. In contrast, discontinuing bDMARDs resulted in a significant risk of loss of remission or low disease activity and an increased risk of radiographic progression [8]. Studies in ankylosing spondylitis (AS) have thus far shown similar findings. Brandt et al. 2003 conducted a study in 30 patients with established AS treated with etanercept, a TNFi [9]. The authors found that after treatment discontinuation, $75 \%$ of patients had experienced a clinical relapse within 6 weeks. In a study by Cantini et al. 2013, 43 patients with established AS who achieved clinical remission were randomised to receive etanercept $50 \mathrm{mg}$ every other week, or etanercept $50 \mathrm{mg}$ weekly [10]. The authors found no significant difference in the percentage of patients in remission between the groups at more than 20 months of follow-up.

This review aims to summarise the current evidence on the tapering and discontinuation of bDMARDs in PsA. We focus on i) requirements, ii) process, iii) outcomes, and iv) predictors of tapering and discontinuation of bDMARDs. We performed a literature search using PubMed, with the following key words: 'biologic', 'TNFi', 'tapering', 'dose-reduction', 'discontinuation', 'withdrawal' and 'psoriatic arthritis'. We also reviewed the references of key papers to ensure relevant studies were included in this review.

\section{Tapering of bDMARDs in PsA}

2.1 Requirements for tapering of bDMARDs 
All studies on bDMARD tapering in PSA have required patients to either be in remission or have low disease activity. To define this, many studies used treat-to-target criteria such as the minimal disease activity criteria (MDA) [11-13], and a few used the DAS28-ESR score. To achieve MDA, patients are required to meet any five of the seven criteria. The seven criteria are: tender joint count $\leq 1$, swollen joint count $\leq 1$, Psoriasis Activity and Severity Index (PASI) $\leq 1$ or body surface area $(B S A) \leq 3$, patient pain visual analogue scale (VAS) $\leq 15$, patient global VAS $\leq 20$, health assessment questionnaire (HAQ) $\leq 0.5$, tender entheseal points $\leq 1$. Of the two studies which used the DAS28-ESR score: one required DAS28-ESR $\leq 3.2$ [14] and the other required DAS28-ESR $\leq 2.6$ [15]. Several studies required patients to be in 'clinical remission' prior to bDMARD tapering [16-18]. Although the precise definition of this term varied, typically patients were required to be free of actively inflamed joints and inflammatory extra-articular features, with normal acute phase reactants.

There is a wide discrepancy between the stringency of the requirements for bDMARD tapering, depending on the criteria used. The DAS28 only takes into account peripheral arthritis, whilst the MDA identifies low disease activity across multiple domains but not remission. In contrast, 'remission' is a very stringent definition that requires a comprehensive set of criteria to be met across multiple domains, and for many patients, it is hard to achieve. Across studies, the length of time patients were required to be in remission or low disease activity was also highly variable, ranging from six months to a year.

\section{2 bDMARD tapering regimen}

There are two ways in which bDMARDs could be tapered: by reducing each dose or by increasing the time interval between doses. As TNFi are the most commonly used bDMARD in PsA, most tapering studies have focused on TNFi tapering. The dose is typically reduced for intravenous TNFi, and the dosing interval increased for subcutaneous TNFi (Table 1).

During the TNFi tapering process, patients are typically maintained on their usual dose of csDMARDs and non-steroidal antiinflammatory drugs although in some cases, these were also tapered [17]. At each follow up, disease activity is assessed through clinical examination and laboratory markers, with some also using additional imaging modalities such as ultrasound. Most studies follow up on patients at 3 and 6 months with less frequent follow up thereafter, as loss of remission is likely to occur soon after TNFi tapering [14]. Between follow-ups, patients are able to contact study coordinators, with the option of being reviewed quickly if they feel their disease is flaring.

Once remission is lost, the tapered bDMARD dose is typically increased to the previous dose, and steroids may be used both to dampen the immune response and for symptomatic relief. There is little standardisation between studies on the criteria that defines 'loss of remission'. Most use a combination of clinical features and laboratory markers [13, 17], several use the loss of fulfilment of composite targets such as the DAS28-ESR or the MDA criteria [14], and one study included patient-defined disease recurrence in the 'loss of remission' criteria [14].

\subsection{Success of bDMARD tapering in PsA}

Most studies on bDMARD tapering in PSA are small retrospective observational studies, from which it is difficult to draw robust conclusions. The current evidence suggests that bDMARD tapering appears to be feasible and safe in PsA, and that it is possible to maintain remission on a tapered dose for a prolonged period of time (Table 2).

In an Italian study of 54 patients with peripheral and axial subtypes of PsA, etanercept tapering occurred via a stepwise algorithm [17]. Patients were started on etanercept $25 \mathrm{mg}$ twice-weekly, and switched onto a weekly regimen if they were in sustained clinical remission at 12 and 16 weeks. At 24 and 28 weeks, if clinical remission persisted despite the increase in 
drug-free interval, patients were switched onto a fortnightly regimen. Of 29 patients who met the criteria for bDMARD tapering, $21(72 \%)$ were able to maintain remission on a tapered dose: $6(21 \%)$ patients were maintained on a weekly regimen, and 15 (51\%) were maintained on a fortnightly regimen. In another Italian study of 141 patients with PsA, the dosing interval could be extended in 65 (46\%) patients who met tapering criteria on adalimumab and etanercept [12]. In all 65 patients, remission was maintained over a 4-year period. These findings were echoed in several other European studies. Cantini et al 2012 conducted a prospective case-control study of 76 patients with PSA [16]. Of these, 53 met criteria for adalimumab tapering, and 47 (89\%) were able to maintain remission over a mean follow up period of $28.9 \pm 8.4$ months. In a UK study of patients with PSA on TNFi, $9(60 \%)$ of the 15 who met the tapering criteria maintained remission for a mean duration of one year [15]. At 6 months, the disease activity scores were similar between those who underwent tapering and those who continued on the standard TNFi dose. Similarly, in a Spanish study of 102 patients with PsA, $23(82 \%)$ of the 28 who met the tapering criteria were maintained on a tapered dose for more than a year without experiencing loss of remission [13]. The authors found no significant difference in the percentage of patients in MDA between those on the tapered doses compared to those on the full bDMARD doses. There was also no significant difference between the ultrasound assessed disease activity of the two groups, suggesting that disease control can be achieved with tapered doses of bDMARD.

In addition to investigating the feasibility of bDMARD tapering, two studies also examined the financial impact. Lorenzin et al 2015 [12] calculated that increasing the drug-free interval for $40 \mathrm{mg}$ adalimumab from every 2 weeks to every 3.12 weeks could result in savings of over $€ 8,000$ per person per year. By increasing the drug-free interval for $25 \mathrm{mg}$ etanercept from twiceweekly to every 2.75 weeks, savings of over $€ 17,000$ could be achieved. In another study of a mixed cohort of 144 patients with RA, AS and PSA receiving either adalimumab or etanercept at a reduced dose for at least 6 months, the authors concluded that more than €1 million was saved for this cohort [18].

\subsection{Predictors of successful bDMARD tapering}

As bDMARD tapering risks loss of remission, there is considerable clinical value in finding robust predictors of the patients most likely to sustain remission on a tapered dose. To date, the literature on this topic is very limited and further high-quality studies are warranted. Factors reported to be associated with an increased likelihood of maintaining remission on a tapered bDMARD dose include lower disease activity and longer duration on TNFi prior to tapering [13, 14]. Concomitant use of csDMARDs did not increase the likelihood of maintaining remission on a tapered bDMARD dose [14].

\section{Discontinuation of bDMARDs in PsA}

\subsection{Requirements for discontinuation of bDMARDs}

Across studies, the criteria for patients with PSA to discontinue bDMARD was highly variable. However, all required the patient to be in either remission or in low disease activity. Criteria used by studies to define this included DAS28-ESR $\leq 2.6$ [19], Clinical Disease Activity Index (CDAI) $\leq 10$ or physician's global assessment of skin psoriasis $\leq 20 / 100$ [20]. Some studies used the term 'clinical remission' which takes into account examination findings, patient-reported symptoms, and laboratory markers. Most studies required patients to be in remission for a minimum of 6 months in order to be eligible for consideration of bDMARD discontinuation.

\section{2 bDMARD discontinuation regimen}

Most studies discontinued bDMARDs immediately in patients who met the criteria. In one study of patients on multiple drugs, the discontinuation process was carried out in phases over 4 to 8 weeks, whereby the last treatment added was the first discontinued [21]. 


\subsection{Success of bDMARD discontinuation}

Current evidence on the outcomes following bDMARD discontinuation is based on a few small studies, and the overall quality of the evidence is poor (Table 3). Findings from two studies suggest that bDMARD discontinuation could be feasible in PsA, with sustained clinical benefit. Cantini et al 2008 conducted a study of 236 patients with peripheral PSA [22], of which 76 were on TNFi. Of these, 35 patients were able to maintain remission after drug discontinuation. Comparing patients with PsA on TNFi to those on methotrexate alone, the frequency of remission after drug discontinuation was much higher when treated with TNFi (79.5\% vs 20.5\%; p<0.001). Interestingly, the duration of remission after drug discontinuation was not significantly different to those receiving continuous treatment (12.0 \pm 2.4 vs $14.0 \pm 8.1$ months), suggesting that drug discontinuation is a feasible option in PsA. In a CORRONA registry study of 5,945 patients with PsA, 352 patients with low disease activity discontinued TNFi, with 302 having follow-up data available [20]. Over half of the patients who discontinued TNFi maintained remission, and the estimated median time to loss of remission was 29.2 months. Of the 146 patients who lost remission, only 10 required re-initiation of TNFi and the rest were able to be managed on cSDMARDs and prednisolone. This suggests that bDMARD discontinuation can offer sustained clinical benefit whilst minimising financial cost and limiting the risks associated with prolonged TNFi exposure.

However, three other studies found a high rate of relapse following bDMARD discontinuation in patients with PsA, suggesting that bDMARD discontinuation carries a significant risk of loss of disease control and should not be undertaken. In a small study of 12 patients with PsA treated with TNFi, consisting of 5 patients on TNFi monotherapy and 7 in combination with methotrexate, TNFi were discontinued at the initial study visit if patients were in continuous remission for $\geq 6$ months [23]. The authors found an unexpectedly high rate of loss of remission following drug discontinuation, with 10 of 12 patients affected. In contrast to Cantini et al 2008 [22], there was no difference between the frequency of loss of remission for patients treated with TNFi compared to those treated with methotrexate. The recurrence of symptoms occurred soon after drug discontinuation, within a mean of $74.5 \pm 51.7$ days, and was characterised by the development of joint symptoms. Similarly, in a retrospective study of etanercept discontinuation in 47 patients with PSA [19], the mean time to loss of remission after drug discontinuation was $18.47 \pm 1.62$ weeks. Compared to the findings of Huynh et al 2017 [20], the remission period was much shorter. Moverley et al 2015 conducted a study of 17 patients with PsA, with 11 patients randomised to drug discontinuation and 6 to standard care [21]. Although this is the only randomised controlled trial included in this review, it was also a feasibility study with small sample sizes due to under-recruitment resulting in the groups not being well matched. The authors found that 6 of 11 patients in the discontinuation group had a loss of remission. Of these, 4 were previously on a bDMARD plus methotrexate, and two were on methotrexate only. A further 4 patients in the discontinuation arm experienced a relapse rate soon after the study's completion at 3 months, suggesting that drug discontinuation carries a substantial risk of loss of remission.

Compared to bDMARD tapering, bDMARD discontinuation in patients with PSA seems to carry a substantially increased risk of loss of remission. There is a large variation in the time to loss of remission following drug discontinuation in the literature, ranging from a few weeks to many months. This is significant because it affects the balance of benefits to risks in deciding treatment options. At present, the evidence is insufficient to guide clinical decision-making and further powered studies are required.

\subsection{Predictors of successful bDMARD discontinuation}

Factors reported with an increased likelihood of loss of remission following drug discontinuation in patients with PSA include: higher disease activity at time of TNFi discontinuation, current smokers, male sex, increased skin involvement, and synovial hypertrophy seen on ultrasound prior to discontinuation [20,23]. The number of previous TNFi, or overweight or obese status did not seem to influence the likelihood of loss of remission [20]. It is unclear whether disease duration affects the likelihood of loss of remission. The CORRONA study reported no association [20], however another study found that longer disease duration increased the likelihood of loss of remission [23]. Further research is required to investigate this. 


\section{Restoring remission after relapse}

As there is a risk of loss of remission following both bDMARD tapering and discontinuation, it is important to know whether remission can be regained. Current evidence suggests that reinstating the bDMARD dose that led to remission previously can help to re-achieve disease control following loss of remission in both bDMARD tapering and discontinuation, although this could take several months.

Cantini et al 2012 found that in patients who had loss of remission following tapering of adalimumab, decreasing the dose interval from $40 \mathrm{mg}$ every month to 2 weeks resulted in remission after a mean interval of $5.1 \pm 2.4$ months [16]. The findings are echoed in another study of patients tapered on etanercept [17]. The authors found that decreasing the dose interval helped to restore remission in those who failed tapering. In a UK study, reinstating the initial dose in those who had loss of remission following tapering enabled low disease activity to be achieved in all 6 patients by 24 weeks [14]. However, compared to those successfully maintained on a tapered TNFi dose, the disease activity in the group who had loss of remission was significantly higher. This suggests that despite regaining remission, it may be difficult for those who had loss of remission to recapture the same degree of disease control compared to those who maintained remission on a tapered dose of bDMARD.

In patients with PSA who experience a loss of remission following drug discontinuation, a study found that reinstating bDMARDs promptly enabled restoration of remission within 3 months in all 10 patients [23]. Similarly, another study found that restarting etanercept following loss of remission post drug discontinuation helped to recapture remission [19]. Notably, the DAS28-ESR, ESR and CRP after 12 weeks of restarting etanercept was comparable to that at the time of etanercept discontinuation, suggesting it is possible to recapture the previous level of disease control.

Where disease control is lost following bDMARD tapering or discontinuation, rather than re-introducing the same bDMARD, another valid therapeutic option is to switch to a different bDMARD $[24,25]$. Current evidence suggests that bDMARD switching may be effective in regaining disease control, although the response to the second TNFi is typically poorer compared to those who persist on the first TNFi. As most of the data is based on patients who switch TNFi due to inefficacy or adverse effects, it is unclear whether this observation applies to patients who respond to the first TNFi, but switch after loss of disease control due tapering or discontinuation.

It remains unclear whether radiographic progression occurs after remission is re-achieved, following loss of remission post bDMARD tapering or discontinuation. Future studies are required to address this.

\section{Current guidelines on tapering and discontinuation of bMDARDs in PsA}

Despite the lack of high-quality data on bDMARD tapering and discontinuation in PSA, guidelines have been developed to help clinicians to standardise regimes and optimise care. The Spanish Rheumatology Society and Hospital Pharmacy Society Consensus recently published a guideline on bDMARD tapering and discontinuation in patients with RA, AS and PsA, with the main recommendations summarised in Table 4 [26]. Notably, they recommend that bDMARD discontinuation should only be considered in patients who successfully maintain sustained remission on a tapered dose and should not be attempted without prior tapering. They also suggest that monitoring plasma levels of bDMARDs could help in deciding which patients should undergo discontinuation, for example, those with undetectable trough bDMARD levels following tapering who remain in sustained remission. 
A follow-on study by the Spanish Rheumatology Society aimed to develop appropriateness criteria for bDMARD tapering in patients with RA, axial spondyloarthritis and peripheral spondyloarthritis [27]. From this work, an online tool has been developed to simplify the decision-making process [28]. Clinicians answer simple questions on disease activity, laboratory markers, duration and time to remission, and use of other DMARDs and steroids. Depending on the disease and the bDMARD, it suggests that tapering is appropriate, uncertain or inappropriate for that patient. Future research will allow these online tools to be further fine-tuned and will likely provide an invaluable resource to both clinicians and patients.

\section{Conclusion}

Current evidence suggest that bDMARD tapering seems to be feasible and safe in patients with PsA who are in remission or with low disease activity. In contrast, discontinuing bDMARDs appears to carry a substantial risk of loss of remission. In those who lose remission, reinstating the standard dose of bDMARD appears to be effective in restoring remission.

At present, there is a paucity of high-quality data on the safety and feasibility of bDMARD tapering and discontinuation in patients with PsA. The available evidence is generally derived from small retrospective observational studies and there is a pressing need for large scale randomised controlled trials to provide more robust data relating to this area to guide clinical decision making. To facilitate this, the poor study recruitment rate needs to be addressed. In a pilot randomised controlled trial on bDMARD discontinuation, only approximately $30 \%$ of the eligible patients were recruited [21]. This was partially because patients tapered or discontinued the dose of their medication prior to formal enrolment, without discussing with their rheumatologist. Moreover, fewer patients than expected fulfilled MDA criteria, emphasising the need for regular objective assessments of disease activity rather than purely relying on patients' subjective assessments. This will enable a treat-to-target approach allowing care and outcomes to be optimised for the maximal number of patients. Another key reason for underrecruitment may be due to patients being unwilling to trial a change in medication regimen when they feel their arthritis is under control, for fear of loss of remission. In this situation, it is important to discuss the risks and benefits for the individual patient so a shared decision can be made.

There is also a need for standardisation between studies, for example regarding criteria for bDMARD tapering or discontinuation, and what constitutes loss of remission. This will facilitate improved comparison between studies and will enable data to be collated in meta-analyses. The MDA is a validated measure to define low disease activity across key domains of PsA and incorporation of this into future studies could help standardisation.

For patients, drug tapering appears to be successful for many and could help to minimise the risk of side effects and opportunistic infections from long-term immunosuppression, reduce the burden associated with frequent drug administration and subsequently improve compliance. For resource-limited healthcare systems, considerable financial savings could be possible, enabling funds to be reallocated elsewhere. These findings must be confirmed with large scale studies, as they have the potential to bring significant benefits both to patients and to resource-limited healthcare systems.

\section{References}

1. Van den Bosch F, Coates L. Clinical management of psoriatic arthritis. The Lancet. 2018 Jun 2;391(10136):2285-94.

2. Gudu T, Kiltz U, de Wit M, Kvien TK, Gossec L. Mapping the Effect of Psoriatic Arthritis Using the International Classification of Functioning, Disability and Health. J Rheumatol. 2017;44:193.

3. Gossec L, Smolen JS, Ramiro S, Wit M de, Cutolo M, Dougados M, et al. European League Against Rheumatism (EULAR) recommendations for the management of psoriatic arthritis with pharmacological therapies: 2015 update. Annals of the Rheumatic Diseases. 2016 Mar 1;75(3):499-510. 
4. NICE [TA199]. Etanercept, infliximab and adalimumab for the treatment of psoriatic arthritis. 2010.

https://www.nice.org.uk/guidance/ta199. Accessed 25 September 2018.

5. Mease PJ, Kivitz AJ, Burch FX, Siegel EL, Cohen SB, Ory P, et al. Etanercept treatment of psoriatic arthritis: Safety, efficacy, and effect on disease progression. Arthritis \& Rheumatism. 2004 50(7):2264-72.

6. Coates LC, Moverley AR, McParland L, Brown S, Navarro-Coy N, O'Dwyer JL, et al. Effect of tight control of inflammation in early psoriatic arthritis (TICOPA): a UK multicentre, open-label, randomised controlled trial. The Lancet. 2015 Dec 19;386(10012):2489-98.

7. van Herwaarden N, den Broeder AA, Jacobs W, van der Maas A, Bijlsma JWJ, van Vollenhoven RF, van den Bemt BJF. Downtitration and discontinuation strategies of tumor necrosis factor-blocking agents for rheumatoid arthritis in patients with low disease activity. Cochrane Database of Systematic Reviews 2014, Issue 9. Art. No.: CD010455. DOI: 10.1002/14651858.CD010455.pub2.

8. Henaux S, Ruyssen-Witrand A, Cantagrel A, Barnetche T, Fautrel B, Filippi N, et al. Risk of losing remission, low disease activity or radiographic progression in case of bDMARD discontinuation or tapering in rheumatoid arthritis: systematic analysis of the literature and meta-analysis. Annals of the Rheumatic Diseases. 2018 Apr 1;77(4):515-22.

9. Brandt J, Khariouzov A, Listing J, Haibel H, Sörensen H, Grassnickel L, et al. Six-month results of a double-blind, placebo-controlled trial of etanercept treatment in patients with active ankylosing spondylitis. Arthritis \& Rheumatism. 48(6):1667-75.

10. Cantini F, Niccoli L, Cassarà E, Kaloudi O, Nannini C. Duration of remission after halving of the etanercept dose in patients with ankylosing spondylitis: a randomized, prospective, long-term, follow-up study. Biologics. 2013;7:1-6

11. Coates LC, Fransen J, Helliwell PS. Defining minimal disease activity in psoriatic arthritis: a proposed objective target for treatment Annals of the Rheumatic Diseases. 2010 Jan 1;69(01):48-53.

12. Lorenzin M, Ortolan A, de Hooge M, Frallonardo P, Piccoli A, Cozzi F, et al. Lengthening the time intervals between doses of biological agents in psoriatic arthritis patients: A single-center retrospective study. Int J Immunopathol Pharmacol. $2015 \mathrm{Dec}$ 1;28(4):479-87.

13. Janta I, Martínez-Estupiñán L, Valor L, Montoro M, Rodriguez OB, Aragüés IH, et al. Comparison between full and tapered dosages of biologic therapies in psoriatic arthritis patients: clinical and ultrasound assessment. Clin Rheumatol. 2015 May 1;34(5):935-42.

14. Fong W, Holroyd C, Davidson B, Armstrong R, Harvey N, Dennison E, et al. The effectiveness of a real life dose reduction strategy for tumour necrosis factor inhibitors in ankylosing spondylitis and psoriatic arthritis. Rheumatology (Oxford). 2016 Oct 1;55(10):1837-42.

15. L. López-Vives, P.V. Estrada, I. Martín-Esteve, M. Aparicio, X. Juanola, J.M. Nolla, J. Rodríguez-Moreno. Dose reduction and/or withdrawal of anti-TN treatment in psoriatic arthritis. Annals of the Rheumatic Diseases 71(Suppl 3):108-109 [abstract]

16. Cantini F, Niccoli L, Cassarà E, Kaloudi $O$, Nannini C. Sustained maintenance of clinical remission after adalimumab dose reduction in patients with early psoriatic arthritis: a long-term follow-up study. Biologics. 2012;6:201-6

17. de Stefano R, Frati E, de Quattro D, de Stefano L. Low Doses of Etanercept Can Be Effective to Maintain Remission in Psoriatic Arthritis Patients: JCR: Journal of Clinical Rheumatology. 2018 Apr;24(3):127-131.

18. J B, Anton R, Navarro Ruiz A, Castera M, Jd R, A M. Persistence and economic impact of etanercept and adalimumab dose reduction in rheumatoid arthritis, psoriatic arthropathy and ankylosing spondylitis patients with at least 1 year in clinical remission: experience from 2 Spanish teaching hospitals during 5 years of follow-up. Value Health 2015; 18: A643. [abstract]

19. Chimenti MS, Esposito M, Giunta A, Graceffa D, Babino G, Teoli M, et al. Remission of Psoriatic Arthritis after Etanercept Discontinuation: Analysis of Patients' Clinical Characteristics Leading to Disease Relapse. Int J Immunopathol Pharmacol. 2013 Jul 1;26(3):833-8.

20. Huynh DH, Boyd TA, Etzel CJ, Cox V, Kremer J, Mease P, et al. Persistence of low disease activity after tumour necrosis factor inhibitor (TNFi) discontinuation in patients with psoriatic arthritis. RMD Open. 2017 Jan 1;3(1):e000395.

21. Moverley A, Coates L, Marzo-Ortega H, Waxman R, Torgerson D, Cocks K, et al. A feasibility study for a randomised controlled trial of treatment withdrawal in psoriatic arthritis (REmoval of treatment for patients in REmission in psoriatic ArThritis (RETREAT (F)). Clin Rheumatol. 2015 Aug 1;34(8):1407-12.

22. Cantini F, Niccoli L, Nannini C, Cassarà E, Pasquetti $P$, Olivieri I, et al. Frequency and duration of clinical remission in patients with peripheral psoriatic arthritis requiring second-line drugs. Rheumatology (Oxford). 2008 Jun 1;47(6):872-6.

23. Araujo EG, Finzel S, Englbrecht M, Schreiber DA, Faustini F, Hueber A, et al. High incidence of disease recurrence after discontinuation of disease-modifying antirheumatic drug treatment in patients with psoriatic arthritis in remission. Annals of the Rheumatic Diseases. 2015 Apr 1;74(4):655-60.

24. Merola JF, Lockshin B, Mody EA. Switching biologics in the treatment of psoriatic arthritis. Semin Arthritis Rheum. 2017;47:29-37.

25. Costa L, Perricone C, Chimenti MS, et al. Switching Between Biological Treatments in Psoriatic Arthritis: A Review of the Evidence. Drugs R D. 2017;17:509-522

26. González-Álvaro I, Martínez-Fernández C, Dorantes-Calderón B, García-Vicuña R, Hernández-Cruz B, Herrero-Ambrosio A, et al. Spanish Rheumatology Society and Hospital Pharmacy Society Consensus on recommendations for biologics optimization in patients with rheumatoid arthritis, ankylosing spondylitis and psoriatic arthritis. Rheumatology (Oxford). 2015 Jul;54(7):1200-9.

27. González-Álvaro I, Blasco AJ, Lázaro P, Sánchez-Piedra C, Almodovar R, Bachiller-Corral J, et al. REDOSER project: optimising biological therapy dose for rheumatoid arthritis and spondyloarthritis patients. Heliyon. 2017 Nov 14;3(11):e00452.

28. ReDOSER calculator. http://redoser.ser.es/\#!/. Accessed 1 Oct 2018. 